\title{
CURRENT DISTRIBUTIONS AND \\ THE DE HAAS-VAN ALPHEN OSCILLATION \\ IN A PLANAR SYSTEM OF HALL ELECTRONS
}

\author{
K. SHIZUYA \\ Yukawa Institute for Theoretical Physics \\ Kyoto University, Kyoto 606-01, Japan
}

\begin{abstract}
The current distribution is studied for a finite-width two-dimensional system of Hall electrons, with a clear distinction drawn between the equilibrium edge current and the Hall current. It is pointed out that both the distribution and direction of the equilibrium edge current change dramatically as the number of electron edge states increases, and that this alternating edge current is another manifestation of the de Haas-van Alphen effect. The Hall-current distribution is substantially different from the edge current distribution, and it is shown numerically that the fast-traveling electrons along the sample edge are not the main carriers of the Hall current.
\end{abstract}




\section{Introduction}

One basic question inherent to the quantum Hall effect 1 (QHE) is where in the sample the current flows. The traditional explanations 25 for the QHE are based on a picture that the Hall current is carried by electrons in the sample bulk and regard local disorder as crucial for the formation of visible conductance plateaus. On the other hand, considerable attention has recently been directed to another picture, $\mathrm{O}$ 具 the edge-state picture of the QHE, where the current is taken to flow in channels along the edges of a sample. Experiments 8 appear to favor interpretation of observed results in terms of the edge channels but little is known about the current distribution in real samples so far, except that some information is obtained from observed potential distributions.10]11 Theoretically the current distribution as well as the potential distribution has been studied in some models, $12-19$ and possible connections between the bulk-state and edge-state pictures of the QHE have been discussed.16, 17

The purpose of the present paper is to study the current-carrying properties of the electrons in the sample bulk and near the edges in detail. In equilibrium a prominent current flow arises along the edges of a Hall sample. It is pointed out that both the distribution and direction of the edge current change dramatically as electron edge states increase in number and that this alternating edge current is another manifestation of the de Haas van Alphen (dHvA) effect. The Hall-current distribution turns out substantially different from the equilibrium current distribution, and it is shown numerically that the fast-traveling electrons along the sample edges, though carrying a large amount of current per electron, are not the main carriers of the Hall current.

In Sec. 2 we study the equilibrium current distribution in a disorder-free Hall sample of finite width. In Sec. 3 we examine the Hall current distribution and discuss the transport properties of the edge current. Sec. 4 is devoted to concluding remarks where the effect of disorder is discussed. 


\section{Current distributions in equilibrium}

Consider electrons confined to an infinitely long strip of width $W$ (or formally, a strip bent into a loop of circumference $\left.L_{x} \gg W\right)$ in the presence of a uniform magnetic field $B$ normal to the plane, described by the Hamiltonian:

$$
H_{0}=\frac{1}{2} \omega\left\{\ell^{2} p_{y}^{2}+\left(1 / \ell^{2}\right)\left(y-y_{0}\right)^{2}\right\}
$$

written in terms of $\omega \equiv e B / m$, the magnetic length $\ell \equiv 1 / \sqrt{e B}$ and $y_{0} \equiv p_{x} /(e B)$; the Landau-gauge vector potential $(-B y, 0)$ has been used to supply a uniform magnetic field. We take explicit account of the two edges $y= \pm W / 2$, where the wave function is bound to vanish.

The eigenstates of $H_{0}$ in the sample bulk are Landau levels with discrete spectrum $\omega\left(n+\frac{1}{2}\right)$ labeled by integers $n=0,1,2, \cdots$, and degenerate in $y_{0}=\ell^{2} p_{x}$; the $y_{0}$ labels the center-of-mass position of each orbiting electron. The eigenfunctions in the presence of sharp edges are still labeled by $N=\left(n, y_{0}\right)$ :

$$
\psi_{N}(x, y)=\left(L_{x}\right)^{-1 / 2} e^{i x p_{x}} \phi_{N}(y)
$$

with $\phi_{N}(y)$ given 6 by the parabolic cylinder functions $20 D_{\nu}\left( \pm \sqrt{2}\left(y-y_{0}\right) / \ell\right)$ for electrons residing near the edges $y=\mp W / 2$. The energy spectrum of each Landau level $n$

$$
\epsilon_{n}\left(y_{0}\right)=\omega\left\{\nu_{n}\left(y_{0}\right)+\frac{1}{2}\right\}
$$

is fixed from the boundary condition $\phi_{N}=0$ at $y= \pm W / 2$. The spectra $\nu_{n}\left(y_{0}\right)$ are determined numerically and are functions of a dimensionless combination $y_{0}^{W} / \ell$ :

$$
\nu_{n}\left(y_{0}\right)=\nu_{n}\left[y_{0}^{W} / \ell\right]
$$

where $y_{0}^{W} \equiv y_{0}+W / 2$ refers to the value of $y_{0}$ measured relative to $y_{0}=-W / 2$ near the "lower" edge; $y_{0}^{W} \equiv y_{0}-W / 2$ near the "upper" edge $y_{0} \sim W / 2$. As shown in Fig. 1, $\nu_{n}\left(y_{0}\right)=\nu_{n}\left(-y_{0}\right)$ rise sharply only in the vicinity of the edges $y_{0} \sim \pm W / 2$, and recover the bulk values $n$ as $y_{0}$ moves to the interior a few magnetic lengths away from the edges:6 for

the lowest $n=0$ level, $0 \leq \nu_{0}<0.003$ for $y_{0}^{W}>2.5 \ell$. Applying the Feynman-Hellman 
theorem to $\partial H_{0} / \partial y_{0}$ reveals that a single electron state $N=\left(n, y_{0}\right)$ has its center-of-mass at position

$$
y_{n}^{\mathrm{cm}} \equiv\langle N|y| N\rangle=y_{0}-\ell^{2} \nu_{n}^{\prime}\left(y_{0}\right)
$$

on the $y$ axis. This $y_{n}^{\mathrm{cm}}$ stays within the interval $[-W / 2, W / 2]$ while $y_{0}=\ell^{2} p_{x}$ can take values outside it for electrons residing near the edges.

The normalized wave functions $\phi_{N}(y)$, taken to be real, are functions of $y-y_{0}$ and $y_{0}^{W}$ :

$$
\phi_{N}(y)=(1 / \sqrt{\ell}) \phi_{n}\left(\left(y-y_{0}\right) / \ell ; y_{0}^{W} / \ell\right)
$$

where we have taken $\phi_{n}$ dimensionless so that its dependence on dimensional quantities $\ell, y_{0}$, etc. is made explicit. Let us denote the spatial distribution of a single electron state $N$ as

$$
\rho_{N}(y) \equiv \rho_{n}\left(y ; y_{0}\right)=\frac{1}{\ell} \phi_{n}\left(\left(y-y_{0}\right) / \ell ; y_{0}^{W} / \ell\right)^{2}
$$

which is uniform in $x$ and which is normalized so that

$$
\int_{-W / 2}^{W / 2} d y \rho_{n}\left(y ; y_{0}\right)=1
$$

The associated current density per electron is written as a product of charge $(-e)$, velocity $\left(p_{x}-e B y\right) / m$ and density $\rho_{n} / L_{x}$ :

$$
j_{x}(y \mid N)=\frac{e \omega}{L_{x}}\left(y-y_{0}\right) \rho_{n}\left(y ; y_{0}\right)
$$

The distributions $\rho_{n}\left(y ; y_{0}\right)$ are highly localized and are nonzero only for $\left|y-y_{0}\right| \leq O(\ell)$. In the sample bulk [i.e., $\left.\left|y_{0}\right|<W / 2-O(\ell)\right]$, in particular, $\rho_{n}$ are even functions of $y-y_{0}$ :

$$
\rho_{n}^{\text {bulk }}\left(y-y_{0}\right)=H_{n}(Y)^{2} e^{-Y^{2}} /\left(\ell 2^{n} n ! \sqrt{\pi}\right)
$$

with $Y \equiv\left(y-y_{0}\right) / \ell$. The profile of $\rho_{n}\left(y ; y_{0}\right)$ gets gradually deformed as $y_{0}$ lies closer to the edge, as shown in Fig. 2 (a) for the $n=0$ level. There the $y_{0}^{W}=3.5 \ell$ electron state, depicted with a solid curve, has $\nu_{0} \approx 10^{-5}$ and thus exhibits essential characteristics of an electron in the bulk; clearly the associated current distribution, depicted with a solid curve in Fig. 2 (b), is characteristic of an orbiting electron and carries no net current. In contrast, 
for an electron lying closer to the edge the cyclotron motion gets deformed, yielding a net current, 䀦 which, in view of Eq. (2.5), is proportional to the gradient of the spectra $\nu_{n}$ :

$$
\int_{-W / 2}^{W / 2} d y j_{x}(y \mid N)=-\frac{e \omega}{L_{x}} \ell^{2} \nu_{n}^{\prime}\left(y_{0}\right) .
$$

The current-carrying property of each electron state $N$ is made manifest by decomposing the current $j_{x}(y \mid N)$ into the circulating component $\propto\left(y-y_{n}^{\mathrm{cm}}\right) \rho_{n}\left(y ; y_{0}\right)$ (that carries no net current) and the traveling component $\propto-\ell^{2} \nu_{n}{ }^{\prime}\left(y_{0}\right) \rho_{n}\left(y ; y_{0}\right)$; see Fig. 2 (c) and (d). It is clearly seen from these figures that the electrons residing near the edge (the edge states) carry a large amount of current. Classically these edge states are visualized as electrons hopping along the sample edges.21 They travel fast (with velocity $v_{x}=\omega \ell^{2} \nu_{n}{ }^{\prime} \sim \pm \omega \ell$ ) in opposite directions at opposite sample edges.

Let us next examine the current distributions filled Landau levels support. We shall focus on the case of well-filled levels close to integer filling. It is convenient to use the Fermi potential $E_{\mathrm{F}} \equiv \omega\left(n_{\mathrm{F}}+1 / 2\right)$ or $n_{\mathrm{F}}$ to specify the filling of each level: For a given $n_{\mathrm{F}}$ Landau levels $n$ with $n \leq n_{\mathrm{F}}$ are populated densely with electron states over the range $\left(y_{0}^{-}\right)_{n}<y_{0}<$ $\left(y_{0}^{+}\right)_{n}$ so that $\nu_{n}\left(\left(y_{0}^{ \pm}\right)_{n}\right)=n_{\mathrm{F}}$; the filling fraction of each level is $f_{n}=\left[\left(y_{0}^{+}\right)_{n}-\left(y_{0}^{-}\right)_{n}\right] / W$ with the number of electrons given by $\left(N_{e}\right)_{n}=(e B / 2 \pi) L_{x} W f_{n}$. The $n_{\mathrm{F}}$ stays constant $n_{\mathrm{F}}=n$ while the $n$th level is gradually filled with the electron states residing in the sample bulk (the bulk states), and it increases as soon as the edge states start to be filled. Such filled states $\left(n, y_{0}\right)$ give rise to the current distribution

$$
j_{x}(y)=\frac{e \omega}{2 \pi \ell^{2}} \sum_{n} \int_{\left(y_{0}^{-}\right)_{n}}^{\left(y_{0}^{+}\right)_{n}} d y_{0}\left(y-y_{0}\right) \rho_{n}\left(y ; y_{0}\right) .
$$

In view of Eq. (2.10), it is clear that $j_{x}(y)=0$ in the sample bulk. Figure 3(a) shows the current distribution near the lower edge $y=-W / 2$ for various values of $n_{\mathrm{F}}$. For $0<n_{\mathrm{F}}<1$ only the $n=0$ level carries current, for $1<n_{\mathrm{F}}<2$ two levels $(n=0,1)$ contribute, and so on.

There are two notable features read from the figure: (1) The current flows along the edge in a channel whose width $\sim O(\ell)$ increases with the number of current-carrying levels. This feature has been well known 
per electron in Eq. (2.11). Somewhat unexpected is the following: (2) Both the spatial distribution and direction of the current change dramatically with $n_{\mathrm{F}}$. For small $n_{\mathrm{F}} \sim 0.1$ the edge current flows predominantly in the negative $x$ direction while a large fraction of current flows in the opposite direction for $n_{\mathrm{F}} \sim 0.9$; the integrated amount of current changes its sign at $n_{\mathrm{F}}=0.5$. Analogous patterns of current flow are also seen for each integer interval $n<n_{\mathrm{F}}<n+1$. The amount of current integrated over one edge oscillates with $n_{\mathrm{F}}$, as shown in Fig. 3(b).

These changing and oscillating current distributions may appear puzzling if one notes Eq. (2.11) alone. There is, however, a simple resolution 21: Associated with each orbiting electron is a circulating current $\propto\left(y-y_{n}^{\mathrm{cm}}\right) \rho_{n}\left(y ; y_{0}\right)$ that produces magnetic moment pointing opposite to the applied magnetic field $B$. For a dense collection of electrons in the sample interior this circulating diamagnetic current is averaged out to vanish locally, but leaves a current circulating along its periphery. Indeed, collecting the circulating current components alone yields a current distribution $j_{x}^{\text {circ }}(y)$ localized near the edge, as shown in Fig. 4 (a) for the $n=0$ level with different $n_{\mathrm{F}}$. Also shown in Fig. 4 (b) is the current distribution $j_{x}^{\operatorname{tr}}(y)$ formed by the traveling current components alone for the same $n=0$ level. They combine to build up the current distributions of Fig. 3(a). It is now clear that the current distribution for $n_{\mathrm{F}}=0.1$ in Fig. 3 (a) primarily derives from the orbital diamagnetic current whereas the edge-driven traveling current dominates in the $n_{\mathrm{F}}=0.9$ case. Note that the edge-driven current works to cancel the orbital diamagnetic current. The cancellation is partial in quantum theory,21 leading to the Landau diamagnetism of electrons for three-dimensional samples.

In the present two-dimensional case it is possible to calculate both the diamagnetic and edge-driven components of the edge current explicitly. As a preliminary step, let us first consider a collection of electron bulk states (of the $n$th level) that fill up a half-infinite interval $y_{0} \geq 0$. They lead to the current distribution

$$
j_{x}^{\text {bulk }}(y)=-\frac{e \omega}{2 \pi \ell^{2}} \int_{-y}^{\infty} d z z \rho_{n}^{\text {bulk }}(z)
$$

localized around $y=0$ with spread $\triangle y=O(\ell)$; for $n=0$ this is given by $-(2 \sqrt{\pi})^{-1} e^{-y^{2} / \ell^{2}}$ times $(e \omega / 2 \pi \ell)$. The integrated amount of this diamagnetic current increases with $n$ : 


$$
\int_{y \sim 0} d y j_{x}^{\text {bulk }}(y)=-\left(n+\frac{1}{2}\right) \frac{e \omega}{2 \pi} .
$$

Consider next a collection of electron states that fill up the interval $\left(y_{0}^{-}\right)_{n} \leq y_{0} \leq \eta$ with $\eta$ lying somewhere far in the sample interior. The circulating current components associated with these states, though carrying no net current, combine to build up two prominent current distributions localized near the edge $y=-W / 2$ and around $y=\eta$, which are equal in net amount of current and opposite in sign. This shows that the diamagnetic component of the current localized near the edge $y=-W / 2$ supports a fixed amount22 equal to $-(e \omega / 2 \pi)(n+1 / 2)$, irrespective of its distribution as well as the shape of edge potentials. In particular, the four different distributions of $j_{x}^{\text {circ }}(y)$ in Fig. 4(a) support the same amount of current equal to $-(e \omega / 4 \pi)$.

On the other hand, the edge states of the $n$th level arise for $n_{\mathrm{F}}>n$ and, as seen from Eq. (2.11), carry the amount of current equal to $(e \omega / 2 \pi)\left(n_{\mathrm{F}}-n\right)$ at the lower edge $y \sim-W / 2$. With the two current components put together the $n$th level alone supports the amount of edge current

$$
J_{n}^{\text {edge }}=\frac{e \omega}{2 \pi}\left(n_{\mathrm{F}}-2 n-1 / 2\right) \quad \text { for } n_{\mathrm{F}} \geq n .
$$

For the integer interval $n \leq n_{\mathrm{F}}<n+1$ the lower $(n+1)$ filled levels combine to support the amount of current

$$
J^{\text {edge }}=\frac{e \omega}{2 \pi}(n+1)\left[n_{\mathrm{F}}-\left(n+\frac{1}{2}\right)\right]
$$

localized near the edge $y=-W / 2$. This current- $n_{\mathrm{F}}$ relation accounts for the alternating edge current of Fig. 3(b), and shows that the (integrated) edge current changes its direction precisely at $n_{\mathrm{F}}=n+1 / 2$.

The alternating edge current of Fig. 3 is intimately connected to an oscillatory variation of magnetic susceptibility of an electron gas with varying magnetic field, known as the de Haas - van Alphen (dHvA) effect.21.23 Indeed, the current $J^{\text {edge }}$, circulating along the sample edge, gives rise to uniform magnetization normal to the sample plane and of magnitude (per unit area) 


$$
M_{z}=\mu_{\mathrm{B}}\left(1 / 2 \pi \ell^{2}\right)(n+1)\left[2\left(n_{\mathrm{F}}-n\right)-1\right]
$$

for $n \leq n_{\mathrm{F}}<n+1$, apart from corrections of $O(\ell / W)$ that vanish as $W \rightarrow \infty$, where $\mu_{\mathrm{B}}=e \omega \ell^{2} / 2=e /(2 m)$ is the Bohr magneton (with effective mass $m$ ).

This result is readily verified by thermodynamic methods as well. The simplest way is to consider how the energy of the present finite-width system,

$$
E=\left(L_{x} / 2 \pi \ell^{2}\right) \sum_{n} \int d y_{0} \omega\left\{\nu_{n}\left[y_{0}^{W} / \ell\right]+1 / 2\right\},
$$

responds to an infinitesimal variation of the magnetic field $B$. Keep $p_{x}=y_{0} / \ell^{2}$ fixed and calculate the magnetization $M_{z}=-\left(1 / W L_{x}\right) \delta E / \delta B$ :

$$
M_{z}=-\frac{\mu_{\mathrm{B}}}{2 \pi \ell^{2}} \sum_{n} \int \frac{d y_{0}}{W}\left\{2 \nu_{n}\left(y_{0}\right)+1-\left(2 y_{0}-y_{0}^{W}\right) \nu_{n}^{\prime}\left(y_{0}\right)\right\} .
$$

Note that $\nu_{n}=n$ in the sample bulk and that $\nu_{n}^{\prime} \neq 0$ only in the edge regions. The $y_{0}$ integral therefore is equal to $\left[2 n+1-2\left(n_{\mathrm{F}}-n\right)\right]$ for each filled level $n$, apart from corrections of $O(\ell / W)$; this result is consistent with Eq. (2.15) and thus recovers Eq. (2.17). It is also enlightening to confirm Eq. (2.19) by a direct calculation of the thermodynamic potential $\Omega(\mu, T ; B)=-T \sum_{i} \ln \left(1+e^{\left(\mu-\epsilon_{i}\right) / k T}\right)$ with $T \rightarrow 0$.

With the spectra $\nu_{n}\left(y_{0}\right)$ determined numerically, it is a simple task to express the magnetization (2.19) as a function of $B$ or the filling factor $f=\sum_{n} f_{n} \propto N_{e} / B$. As seen from Fig. 5, the magnetization per electron, $m_{z} \equiv M_{z} /\left(N_{e} / W L_{x}\right)$, plotted as a function of $f$ shows an oscillatory variation characteristic of the dHvA effect. As usual, the gradual variation of $m_{z}$ in each integer interval $n<f<n+1-O(\ell / W)$ is ascribed to the diamagnetism of orbiting electrons in the bulk. In each tiny interval $n+1-O(\ell / W)<f<n+1, m_{z}$ makes a drastic change from diamagnetism to paramagnetism that is caused by the edge states of the $n$th level.

Note here that the electron edge states, because of their tiny filling fraction $O(\ell / W)$, scarcely contribute to the internal energy $E$. Thus, even without explicit account of the edge states, one can still arrive at the dHvA effect by thermodynamic methods (i.e., through $E$ or $\Omega$ ), except that $M_{z}$ now shows discontinuous jumps at integer filling $f=n$. The edge states are invisible in such derivations but are certainly present physically: Note that magnetization 
by nature is continuous as a function of $B / N_{e}$, as seen clearly from the $W=30 \ell$ case of Fig. 5. In view of this continuity, the prominent sign change of $m_{z}$ near integer filling $f=n$ (which can be derived by thermodynamics without the edge states) does imply the presence of the edge states and, in particular, the alternation in direction of the edge current. In this sense, the alternating edge current in Fig. 3 is another aspect of the dHvA effect.

The alternation of the edge current is caused by competition between the circulating and edge-driven components of the current near the edge. The edge-driven component is naturally very sensitive to the shape of the edge potential while the circulating component is not; see Fig. 2 (c) and (d). Correspondingly the edge current in general varies in distribution according to the shape of edge potentials. Still, in net amount per edge, both current components remain unaltered so that Eqs. (2.15) and (2.16) hold, irrespective of the details of edge potentials.

\section{Hall-current distributions}

The alternation of the edge current, which we have just seen, does not necessarily imply that the information the edge current carries also changes the direction of propagation. In this section we clarify this point by studying how the edge current responds to a Hall field.

For simplicity let us consider a uniform field $E_{y}=-\partial_{y} A_{0}(y)$. Its effect is readily taken care of by making the shift

$$
y_{0} \rightarrow \bar{y}_{0} \equiv y_{0}-\left(e \ell^{2} / \omega\right) E_{y}
$$

in $H_{0}$ of Eq. (2.1), and the full Hamiltonian $H_{0}-e A_{0}(y)$ leads to the new spectrum $\epsilon_{n}\left(\bar{y}_{0}\right)+e E_{y} \bar{y}_{0}+O\left(E_{y}^{2}\right)$. The normalized wave function $\phi_{N}(y)$ is given by Eq. (2.6) with the replacement $y_{0} \rightarrow \bar{y}_{0}$, and the current per electron $j_{x}(y \mid N)$ by Eq. (2.9) with $\rho_{n}\left(y ; y_{0}\right) \rightarrow \rho_{n}\left(y ; \bar{y}_{0}\right)$. The current distribution $j_{x}(y \mid N)$ itself barely changes thereby because, under conditions of practical interest, the deviation $\bar{y}_{0}-y_{0}$ is negligibly small.

Actually the Hall current we are interested in is the small deviation,

$$
\triangle j_{x}(y \mid N)=\frac{e \omega}{L_{x}}\left(y-y_{0}\right)\left\{\rho_{n}\left(y ; \bar{y}_{0}\right)-\rho_{n}\left(y ; y_{0}\right)\right\}
$$


representing the response to an applied field. As seen from Fig. 6(a), unlike $j_{x}(y \mid N)$, the Hall current per electron $\triangle j_{x}(y \mid N)$ is primarily composed of traveling components. Here we see that, while the edge states and bulk states are drastically different in the amount of current they carry, they are essentially the same in the Hall-current transport. Numerically an edge state supports even a smaller amount of Hall current than a bulk state, as seen from Fig. 6(b), where the numerically-integrated amount of Hall current per electron, $\triangle J_{x} \equiv \int d y \triangle j_{x}(y \mid N)$, is compared with the net current per electron, $J_{x} \equiv \int d y j_{x}(y \mid N) \propto-\nu_{n}{ }^{\prime}\left(y_{0}\right)$.

It is possible to understand the current-carrying properties of each electron state in a more general way: Of the current $j_{x}(y \mid N)$, the circulating component $\propto\left(y-y_{n}^{\mathrm{cm}}\right) \rho_{n}$ carries no net current. It is the traveling component, associated with the drift of a Hall electron with velocity

$$
\omega \ell^{2} \nu_{n}^{\prime}\left(\bar{y}_{0}\right)+E_{y} / B
$$

that carries a net current (and hence information with it). Accordingly disturbances caused upon a sample, e.g., by varying a magnetic field or electron population will propagate in a direction fixed by the edge with velocity $\omega \ell$. In contrast, the effect of a Hall field (i.e., the Hall current) propagates in a direction fixed by the polarity of $E_{y}$ with velocity $\sim E_{y} / B$. [Numerically $\omega \ell \sim 10^{7} \mathrm{~cm} / \mathrm{s}$ for typical values $\omega \sim 10 \mathrm{meV}$ and $\ell \sim 100 \AA$ while $E_{y} / B \sim 10^{3} \mathrm{~cm} / \mathrm{s}$ for $E_{y}=1 \mathrm{~V} / \mathrm{cm}$ and $B=5 \mathrm{~T}$.] It follows from Eq. (3.3) that the net amount of Hall current per electron is proportional to $1-\ell^{2} \nu_{0}^{\prime \prime}\left(y_{0}\right)$, which reproduces the numerical result in Fig. 6(b) very accurately.

Filled Landau levels support the prominent edge-current distributions of Fig. 3(a), which remain essentially unchanged in the presence of a Hall field as well. These edge currents flow in opposite directions at the two opposite edges of a sample, and in equilibrium with $E_{y}=0$ they combine to vanish. 5 .6 When a Hall field $E_{y}$ is turned on, the Hall current emerges as a small deviation from the equilibrium distribution, as shown in Fig. 6(c) for the present impurity-free and uniform- $E_{y}$ case. It is clear from Fig. 6, contrary to some tempting ideal, that the electron edge states, because of their tiny filling fraction $\sim O(\ell / W)$, share only a tiny portion of the Hall current. 17 


\section{Concluding remarks}

In this paper we have examined current distributions in a Hall bar in the regime of the integer QHE, and shown, in particular, that the edge current changes its distribution and direction as the number of electron edge states increases. This dramatic change is a consequence of competition between the circulating diamagnetic component and edgedriven traveling component of the current carried by electrons near the sample edge, and is closely related to the dHvA effect. It should be emphasized that the dHvA oscillation of magnetization is indirect evidence for the edge states; it is clear physically that without them no oscillation would arise, since orbital magnetization alone leads to diamagnetism.21 In this connection we have seen explicitly that magnetization is continuous as a function of $B / N_{e}$ when the edge states are properly taken into account. We have also seen that the Hall current flows in a manner quite independent of the equilibrium edge-current distribution.

Finally we would like to comment on the influence of disorder. Rapid motions of Hall electrons like cyclotron motion and acceleration by the edge are potentially not very sensitive to disorder. The edge current of Fig. 3, resulting from such rapid motions, will therefore remain prominent in the presence of weak disorder as well, and continue to alternate in direction as the number of edge states increases. In contrast, disorder will substantially modify the current carried by slowly-drifting electrons in the sample bulk: In the presence of disorder a large fraction of electron bulk states get localized and cease to carry current; at the same time, the surviving extended states carry more current and achieve exact compensation. 目, This exact current compensation is a consequence of electromagnetic gauge invariance and takes place under general circumstances involving both bulk and edge states.17

Note now that, of the electron bulk states, those residing near the edge of the sample bulk ("bulk edge"), though influenced by disorder, would have a better chance of staying extended than those far in the bulk. In view of current compensation, a considerable portion of the Hall current, redistributed via disorder, would therefore flow along the sample edges. In this way it has been pointed out 17 that there are two kinds, fast and slow, of edge current in Hall samples in the regime of the integer QHE. 
These two kinds of edge current differ in channel width and in direction of flow. The fast component is nothing but the alternating edge current discussed so far, consisting of the two (circulating and traveling) subcomponents; it has a channel width of $O(\ell)$ and flows in opposite directions at opposite sample edges. In contrast, the slow component, the "bulk-edge" Hall current, will have a channel width, related to some localization length characteristic to the bulk edge, which could well be larger than $O(\ell)$. This slow edge current will flow in the same direction at opposite edges and reverse direction when the polarity of the Hall field is flipped; an observed Hall-potential distribution 11 appears to be in support of this feature.

A numerical experiment is now under way to study the current distribution for small samples with randomly distributed impurities, and is yielding results that appear to confirm the effect of disorder on the current distribution described above; details will be reported elsewhere.

\section{Acknowledgments}

The author wishes to thank B. Sakita and Y. Nagaoka for useful discussions. This work is supported in part by a Grant-in-Aid for Scientific Research from the Ministry of Education of Japan, Science and Culture (No. 07640398). 


\section{REFERENCES}

${ }^{1}$ K. von Klitzing, G. Dorda and M. Pepper, Phys. Rev. Lett. 45, 494 (1980). For a review see, The Quantum Hall Effect, edited by R.E. Prange and S.M. Girvin (Springer Verlag, Berlin, 1987).

${ }^{2}$ H. Aoki and T. Ando, Solid State Commun. 38, 1079 (1981).

${ }^{3}$ R. E. Prange, Phys. Rev. B23, 4802 (1981).

${ }^{4}$ R. B. Laughlin, Phys. Rev. B23, 5632 (1981).

${ }^{5}$ B. I. Halperin, Phys. Rev. B25, 2185 (1982).

${ }^{6}$ A. H. MacDonald, and P. Streda, Phys. Rev. B29, 1616 (1984).

${ }^{7}$ P. Streda, J. Kucera and A. H. MacDonald, Phys. Rev. Lett. 59, 1973 (1987); J. K. Jain and S. A. Kivelson, Phys. Rev. B37, 4276 (1988).

${ }^{8}$ M. Büttiker, Phys. Rev. B38, 9375 (1988).

${ }^{9}$ S. Washburn, A. B. Fowler, H. Schmidt, and D. Kern, Phys. Rev. Lett. 61, 2801 (1988); B. J. van Wees et al., Phys. Rev. Lett. 62, 1181 (1989); B. W. Alphenaar, P. L. McEuen, R. G. Wheeler and R. N. Sacks, Phys. Rev. Lett. 64, 677 (1990).

${ }^{10}$ F. Fang and S. Stiles, Phys. Rev. B29, 3747 (1984); H. Z. Zheng, D. C. Tsui, A. M. Chang, Phys. Rev. B32, 5506 (1985); E. K. Sichel, H. H. Sample and J. P. Salerno, Phys. Rev. B32, 6975 (1985).

${ }^{11}$ P. F. Fontein, et al., Phys. Rev. B43, 12090 (1991).

${ }^{12}$ A. H. MacDonald, T. M. Rice, and W. F. Brinkman, Phys. Rev. B28, 3648 (1983).

${ }^{13}$ O. Heinonen and P. L. Taylor, Phys. Rev. B32, 633 (1985).

${ }^{14}$ T. Otsuki and Y. Ono, J. Phys. Soc. Jpn. 58, 2482 (1989).

${ }^{15}$ D. B. Chklovskii, B. I. Shklovskii, and L. I. Glazman, Phys. Rev. B46, 4026 (1992). 
${ }^{16}$ D. J. Thouless, Phys. Rev. Lett. 71, 1879 (1993); C. Wexler and D. J. Thouless, Phys. Rev. B49, 4815 (1994).

${ }^{17}$ K. Shizuya, Phys. Rev. Lett. 73, 2907 (1994); Phys. Rev. B45, 11143 (1992).

${ }^{18}$ Y. Avishai, Y. Hatsugai, and M. Kohmoto, Phys. Rev. B47, 9501 (1993); Y. Avishai and M. Kohmoto, Int. J. Mod. Phys. B9, 2949 (1995).

${ }^{19}$ M. R. Geller and G. Vignale, Phys. Rev. B52, 14137 (1995).

${ }^{20}$ E. T. Whittaker and G. N. Watson, A Course of Modern Analysis, (Cambridge University Press, London, 1927).

${ }^{21}$ L. Landau, Z. Phys. 64, 629 (1930); E. Teller, Z. Phys. 67, 311 (1931); R. E. Peierls, Surprises in Theoretical Physics, (Princeton Univ. Press, Princeton, 1979). The author wishes to thank M. Stone for informing him of Teller's paper.

${ }^{22}$ This value $(e \omega / 2 \pi)(n+1 / 2)$ for the edge current was derived earlier in Ref. 19 within a derivative expansion for a slowly-varying confining potential.

${ }^{23}$ For the dHvA effect in two dimensions, see A. Ishihara, Solid State Phys. 42, 271 (1989); D. Yoshioka and H. Fukuyama, J. Phys. Soc. Jpn. 61, 2368 (1992). 


\section{FIGURES}

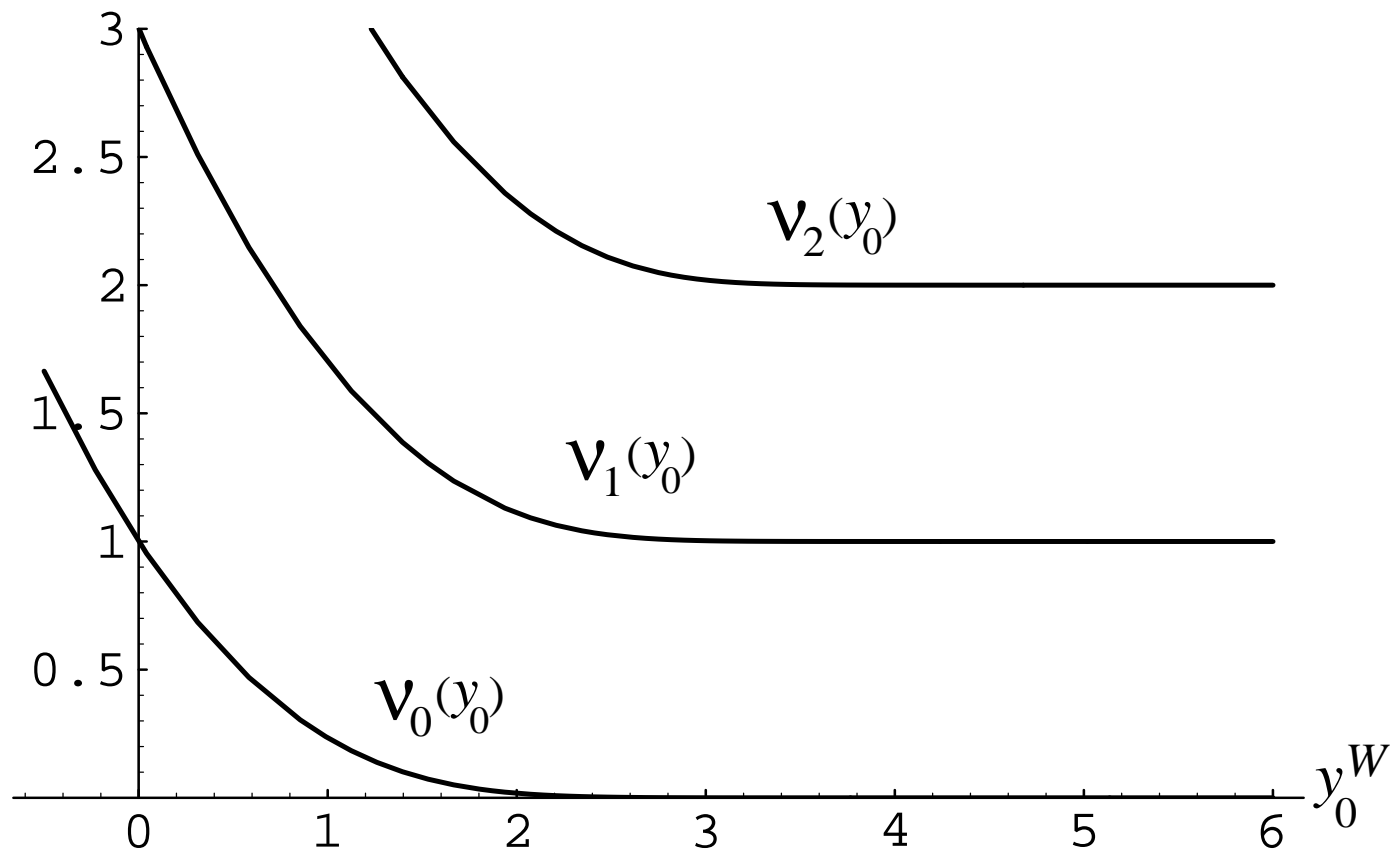

FIG. 1. Spectra $\nu_{n}\left(y_{0}\right)$ plotted as a function of $y_{0}^{W} \equiv y_{0}+W / 2$ (measured in units of the magnetic length $\ell$ ) near the lower edge $y_{0}^{W} \approx 0$. 

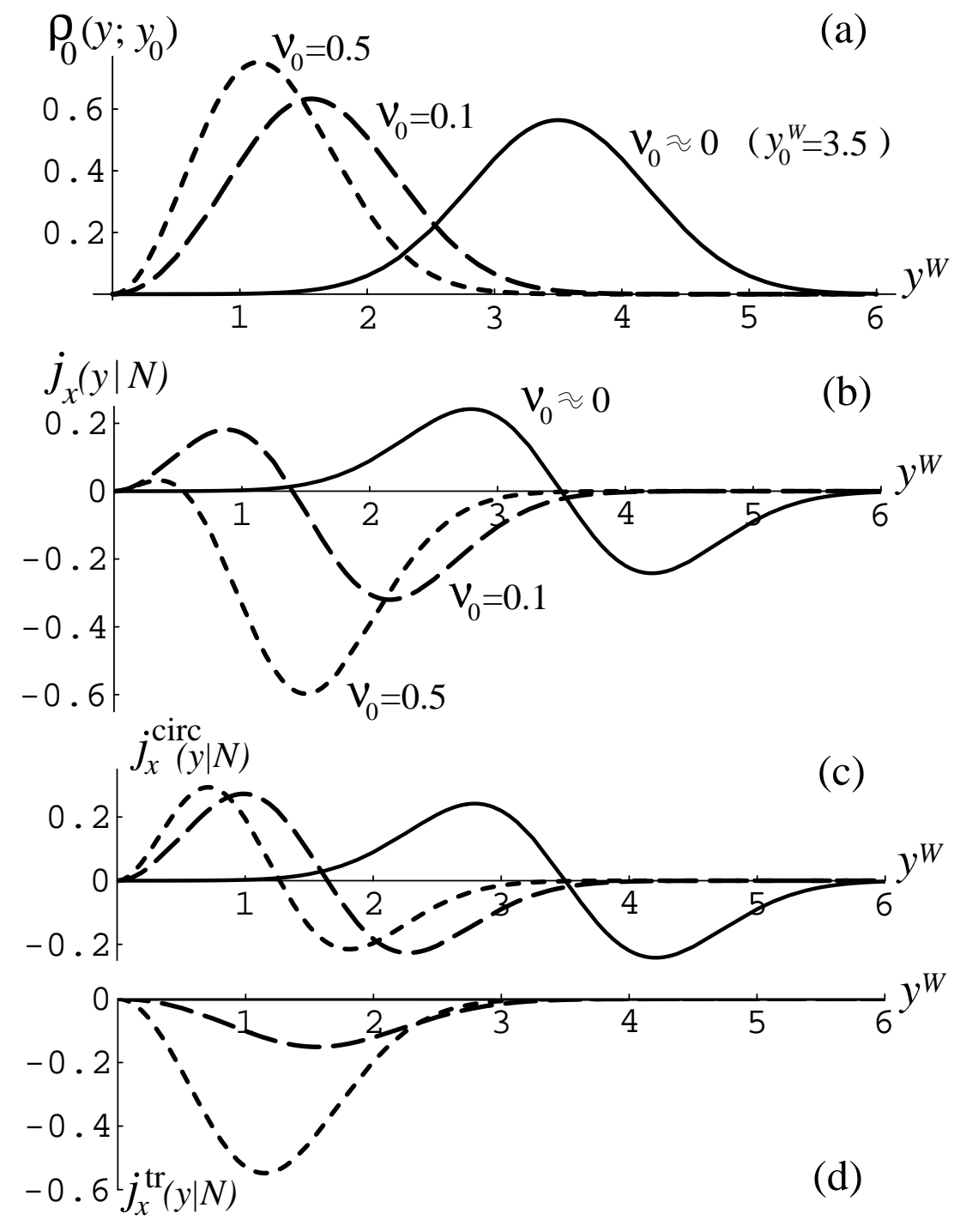

FIG. 2. (a) Spatial distributions $\rho_{n}\left(y ; y_{0}\right)$ of single electron states with different $\nu_{0}\left(y_{0}\right)$ in the $n=0$ level. The solid curve represents the $\nu_{0} \approx 10^{-5}$ electron state with $y_{0}^{W}=3.5 \ell$, the long-dashed curve the $\nu_{0}=0.1$ state with $y_{0}^{W} \approx 1.40 \ell$, and the dashed curve the $\nu_{0}=0.5$ state with $y_{0}^{W} \approx 0.541 \ell$. The coordinate $y^{W}=y+W / 2$, defined relative to the edge $y=-W / 2$, is measured in units of $\ell$. (b) Distributions of the associated current per electron. Here, for convenience, the current $j_{x}(y \mid N)$ is plotted in units of $-e \omega / L_{x}$ so that its sign refers to that of the velocity $v_{x}$ along the $x$ axis. (c) Decomposition of $j_{x}(y \mid N)$ into the circulating component $j_{x}^{\text {circ }}(y \mid N)$ and the traveling component $j_{x}^{\text {tr }}(y \mid N)$. 

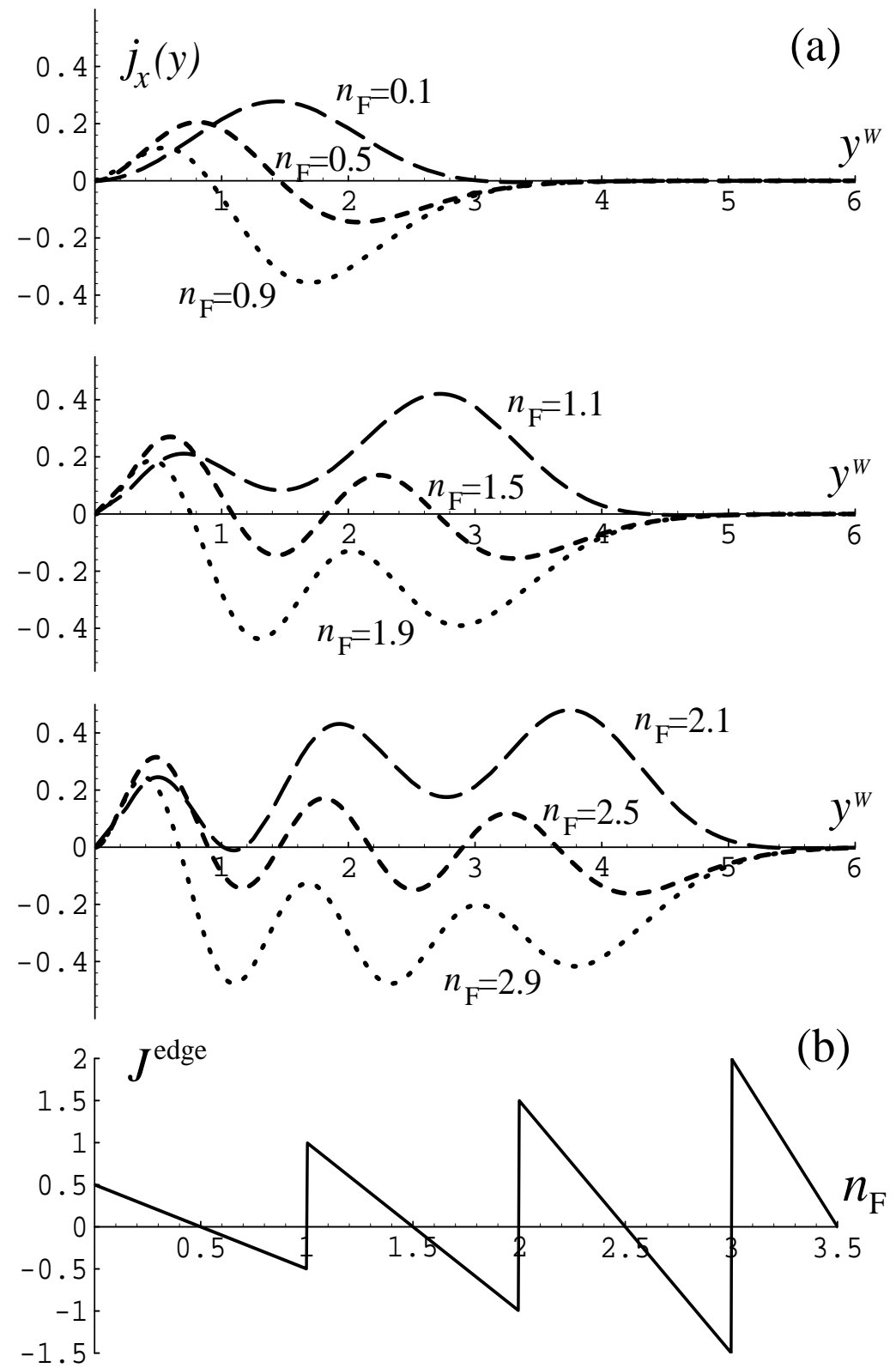

FIG. 3. (a) Current distributions filled Landau levels support near the sample edge $y=-W / 2$; $n_{\mathrm{F}}=E_{\mathrm{F}} / \omega-1 / 2$ refers to the Fermi potential $E_{\mathrm{F}}$. The current $j_{x}(y)$ is measured in units of $-e \omega /(2 \pi)$ and the coordinate $y^{W}=y+W / 2$ in units of $\ell$. (b) The integrated amount of the current per edge [in units of $-e \omega /(2 \pi)]$ oscillates with $n_{\mathrm{F}}$. 


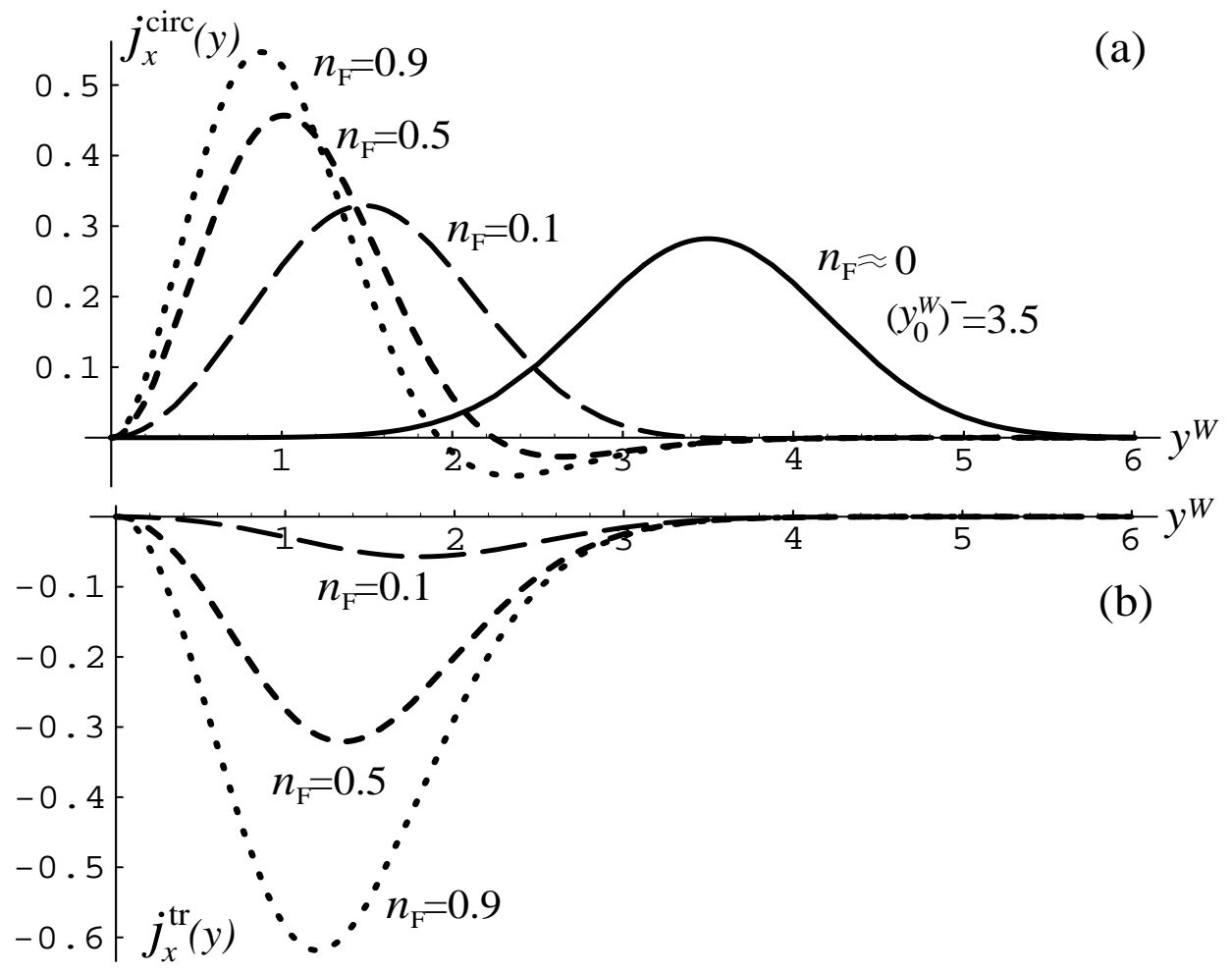

FIG. 4. (a) Current distributions near the edge $y=-W / 2$ built up by the circulating current components alone for the $n=0$ level with different values of $n_{\mathrm{F}}$. The $n_{\mathrm{F}} \approx 10^{-5}$ case refers to the $n=0$ level filled with electron states $y_{0}^{W} \geq 3.5 \ell$. The current is measured in units of $-e \omega /(2 \pi)$, and the coordinate $y^{W}$ in units of $\ell$. (b) Current distributions formed by the traveling current components alone for the same $n=0$ level. 


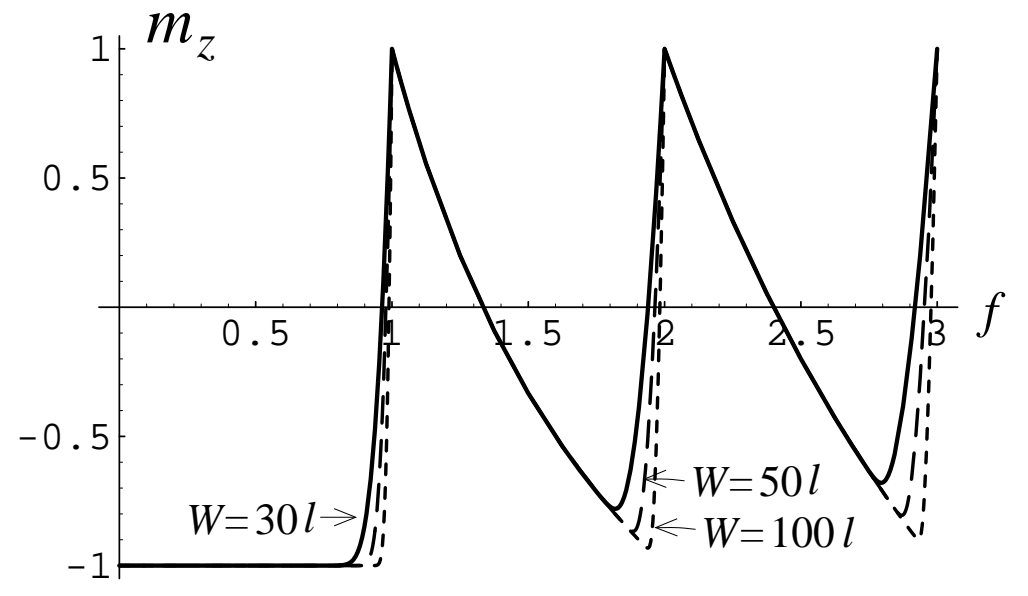

FIG. 5. Magnetization per electron [in units of $\left.\mu_{\mathrm{B}} / 2 \pi \ell^{2}\right]$ v.s. the filling factor $f$ for a Hall bar of finite width $W=30 \ell, 50 \ell$, and $100 \ell$. 

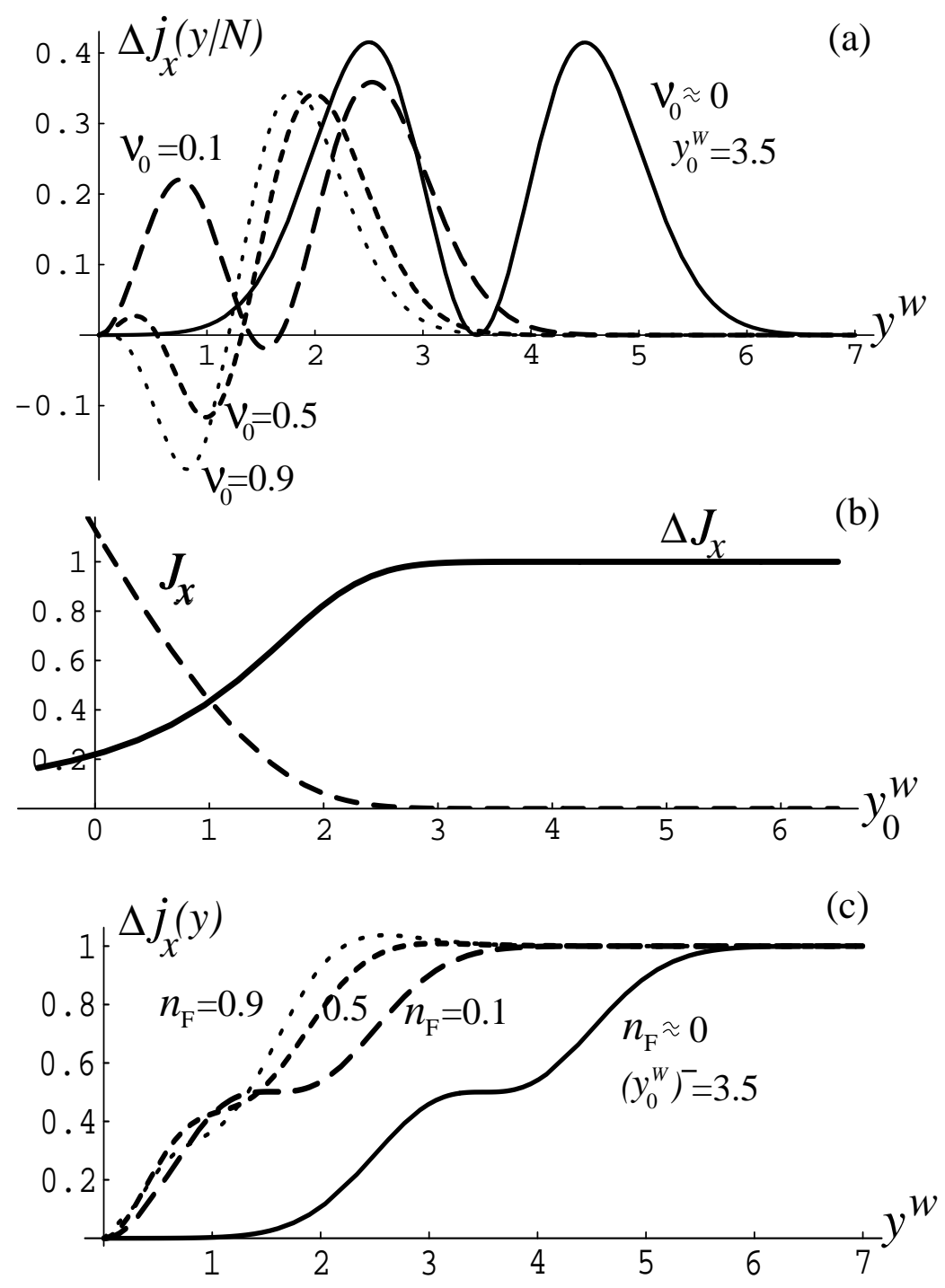

FIG. 6. (a) Distributions of the Hall current per electron, $\triangle j_{x}(y \mid N)$, for electron states with different $\nu_{0}\left(y_{0}\right)$ in the $n=0$ level. The current is plotted in units of $10^{-3} \times\left(-e \omega / L_{x}\right)$ for $e \ell E_{y} / \omega=10^{-3}$. (b) The Hall current per electron $\triangle J_{x}=\int d y \triangle j_{x}(y \mid N)$ (in units of $\left.-e^{2} \ell^{2} E_{y} / L_{x}\right)$ for electron states in the $n=0$ level, in comparoson with the net current per electron $J_{x}=\int d y j_{x}(y \mid N)$ (in units of $\left.e \omega \ell / L_{x}\right)$. (c) Hall-current distributions near the edge for the $n=0$ level. The edge states increase in number with $n_{\mathrm{F}}$. 